\title{
The low incidence of clinically significant heart disease in school-age children following COVID-19
}

\author{
Adam W. Powell ${ }^{a}$ (D), Christopher J. Statile ${ }^{a}$ (D), Nicolas L. Madsen ${ }^{a}$, Allison A. Divanovic ${ }^{a}$, Sean M. Lang ${ }^{a}$ (D)
}

\begin{abstract}
The burden of clinically-apparent cardiac injury secondary to coronavirus disease 2019 (COVID-19) in school-age children is unclear. Over 12 months at a large academic pediatric hospital in the Midwestern portion of the United States, there were 1481 COVID-19 positive non-hospitalized otherwise healthy schoolaged children with 195 having cardiac testing performed. While incidental findings occurred, no definitive COVID-19 related cardiac pathology was discovered. Additionally, only $\sim 3 \%$ of children had acute cardiac symptoms necessitating evaluation by pediatric cardiology. School-age children who were not hospitalized for COVID-19 have a very low risk of having clinically significant cardiac damage and are more likely to discover incidental findings.

Key words: COVID-19, hearth diseases, sequelae, post-acute COVID-19 syndrome.
\end{abstract}

http:/ / dx.doi.org/10.5546/ aap.2022.eng.54

To cite: Powell AW, Statile CJ, Madsen NL, Divanovic AA, Lang SM. The low incidence of clinically significant heart disease in school-age children following COVID-19. Arch Argent Pediatr 2022;120(1):54-57.

\section{INTRODUCTION}

Clinically significant cardiac injury is a feared complication in children following coronavirus disease 2019 (COVID-19) infection with these concerns spurring sport cancelations and formation of multiple return to play (RTP) guidelines. ${ }^{1}$ While the prevalence of clinically significant cardiac injury following

a. Department of Pediatrics, University of Cincinnati College of Medicine, Cincinnati, $\mathrm{OH}$.

The Heart Institute, Cincinnati Children's Hospital Medical Center, Cincinnati, $\mathrm{OH}$.

E-mail address:

Adam W. Powell: Adam.Powell@cchmc.org

Funding: None.

Conflict of interest: None.

Received: 7-13-2021

Accepted: 9-2-2021
COVID-19 (defined as myocarditis, myocardial inflammation and / or dysfunction) appears to be low in non-hospitalized competitive college athletes, clinically significant cardiac damage in non-hospitalized school-age children has been inadequately studied..$^{2,3}$ Our objective was to further characterize clinically significant cardiac injury seen in non-hospitalized schoolage children following acute COVID-19 infection.

\section{METHODS}

We performed a retrospective chart review of all non-hospitalized school-age patients (5-18 years-old) who had a positive test for COVID-19 performed at a large academic pediatric hospital in the Midwestern section of the United States or were evaluated by pediatric cardiology for a positive COVID-19 study at an outside facility from March 1, 2020 to February 28, 2021. To limit the study to patients with manifestations of acute COVID-19 infection and to exclude patients with long-term COVID-19 symptoms, patients evaluated $>2$ months following their positive test were excluded. Children with co-morbid chronic disease and multisystem inflammatory condition in children (MIS-C) were excluded. Results from cardiac testing were noted.

This study was approved by the Hospital's Institutional Review Board.

\section{RESULTS}

A total of 1481 otherwise healthy COVID-19 positive school-age patients were included in this study (1413 positives at the hospital; 69 positives outside the hospital) (Figure 1). There were zero deaths in this cohort. Of the 117 patients who saw cardiology, $44 \%$ (52/117) were referred for symptoms. Among the cohort 13\% (195/1481) of school-age, non-hospitalized COVID-19 patients had cardiology testing performed including electrocardiogram, echocardiogram, serial serum troponin levels, and cardiac MRI. Results of cardiac testing are described in Table 1. There were zero cases of definitive COVID-19 related pathology (myocarditis, myocardial inflammation and/or dysfunction) discovered. 
Notable findings are shown in Figure 1. Of note, one patient had an initial echocardiogram concerning for left coronary artery dilation, but subsequent cardiac CT demonstrated normal cardiac dimensions. The initial echocardiogram was felt to be a false-positive as cardiac CT is a more specific instrument to measure coronary arteries. This patient never underwent treatment (i.e. IVIG, aspirin) and was discharged from cardiology once the coronary arteries were demonstrated to be normal.

\section{DISCUSSION}

Among all non-hospitalized children for COVID-19, there were zero cases of definitive COVID-19 related cardiac pathology discovered. Rather the majority of pathology discovered were either incidental findings (bicuspid aortic valve, premature ventricular contractions) or falsepositive results (coronary artery "abnormalities" on echocardiogram with subsequently normal cardiac CT scan). Additionally, few COVID-19 positive school children $(\sim 3 \%)$ have acute symptoms that ultimately necessitate cardiology evaluation. Cardiology referral patterns are mostly driven by adherence to RTP guidelines, including concerns for screening electrocardiogram abnormalities. ${ }^{4}$

Moulson and colleagues performed a large prospective observational cohort study comprising 42 universities and over 3,000 collegelevel athletes who underwent cardiac evaluation. That study found $<1 \%$ of COVID positive athletes demonstrated cardiac involvement with no adverse COVID-related cardiac events. ${ }^{2}$ In our younger cohort, the similar lack of COVIDinduced cardiac findings is not surprising given that younger pediatric patients have lower rates of significant acute symptoms. ${ }^{5}$ However, this has not stopped concern from pediatric

TABLE 1. Cardiac testing for the entire cohort

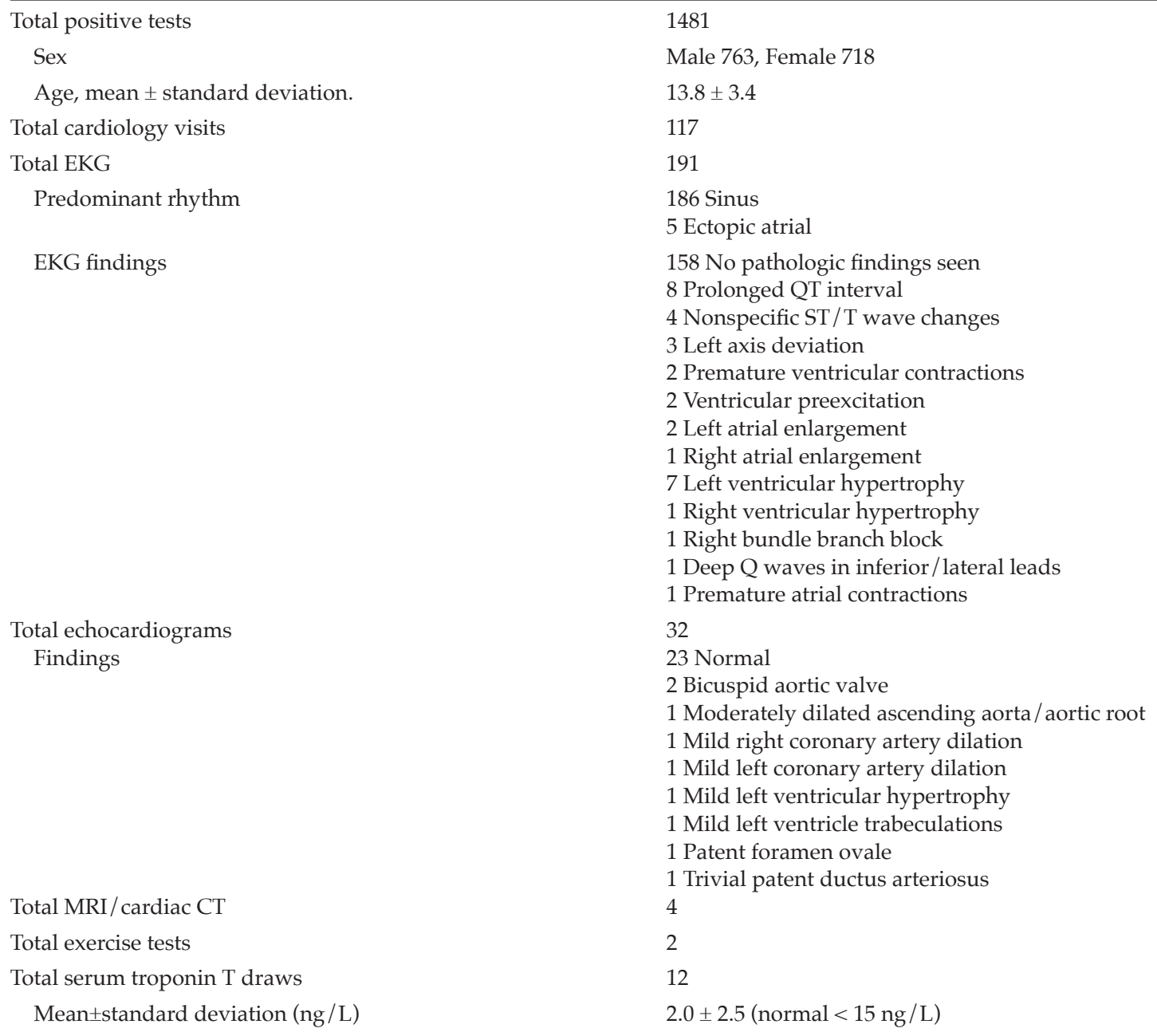


clinical providers and families about the safety of returning to physical activity. Some current RTP guidance may be overly conservative in recommending assessment regardless of infection severity. ${ }^{1}$ In fact, as our results demonstrate, these diagnostic cardiac tests expose patients to the discovery of incidental rather than COVID-related findings, which may lead to increased resource overutilization.
The low prevalence of cardiac injury in this and other studies imply that concerns for cardiac damage should not drive decisions on whether or not to resume in-person schooling or youth sports. These decisions should be driven by public health considerations and reduction of disease spread. The low likelihood of COVIDrelated cardiac involvement suggests a focus on secondary prevention would be more effective,

FIGURE 1. Flowchart of positive COVID-19 patients 5-18 years of age and the distribution of cardiac testing and evaluations in this cohort. Notable findings on cardiac evaluation are shown below

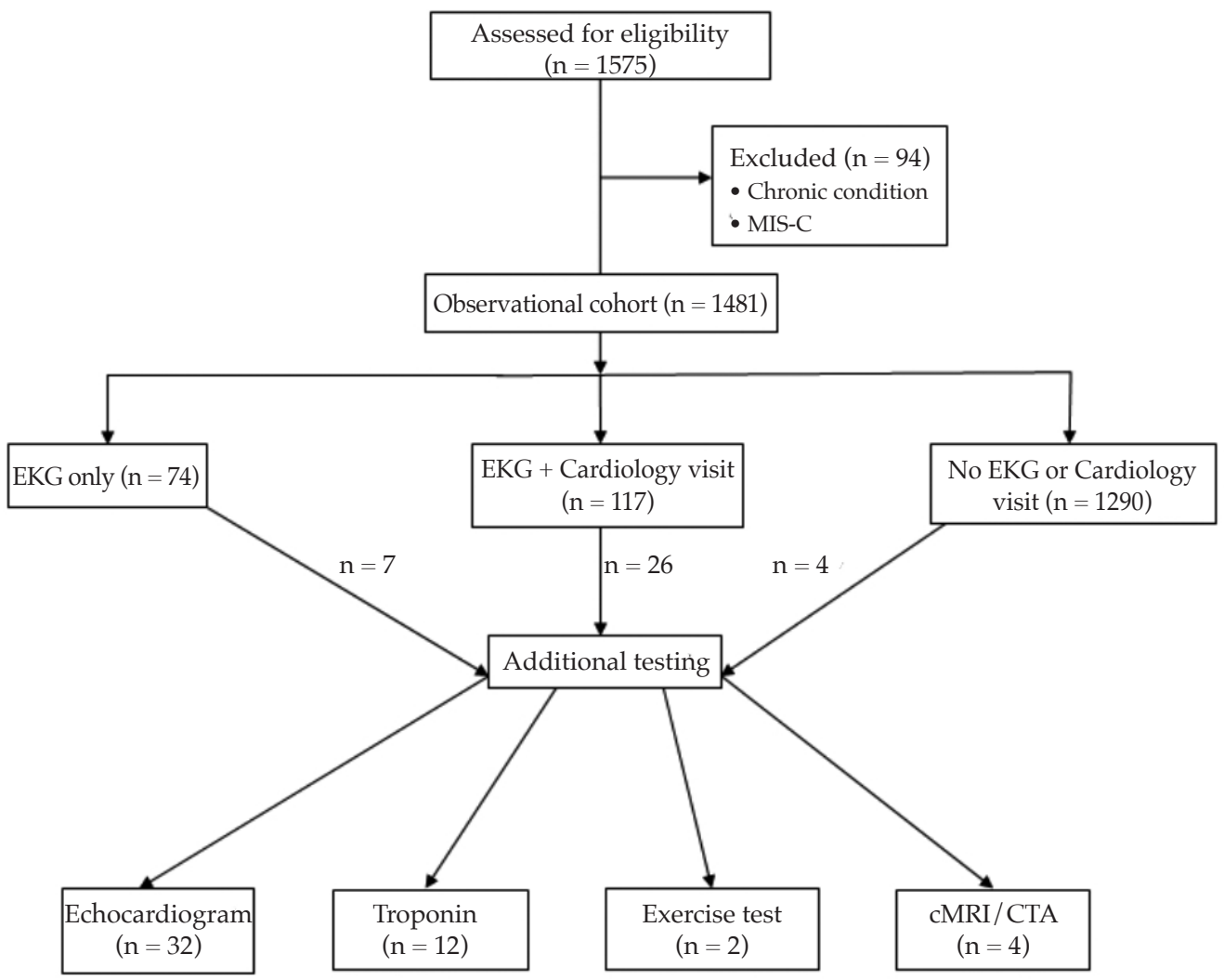

Patient examples:

1. 15 years old female, mildly dilated left main coronary on echocardiogram, subsequently normal on cardiac CT scan performed two weeks later, no follow-up.

2. 18 years old male, concern for anomalous origin right coronary artery on echocardiogram, normal on cardiac CT scan, no followup.

3. 14 years old male, abnormal $\mathrm{T}$ waves on inferolateral leads on EKG, normalized on exercise testing, mild left ventricular hypertrophy on echocardiogram and cMRI, follow-up scheduled for surveillance of mild left ventricular hypertrophy.

4. 17 years old male, mildly dilated right and left ventricles with prominent left ventricular trabeculations on cMRI, follow-up scheduled for surveillance of left ventricular trabeculations.

5. 16 years old male, frequent premature ventricular contractions on EKG, normal echocardiogram, suppression of ventricular ectopy during exercise testing, felt to be unrelated to COVID-19, follow-up in 6 months.

6. Two patients with bicuspid aortic valve, one patient with trivial patent ductus arteriosus, incidental findings. 
such as ensuring coaches and school staff are trained in CPR and expanded automated external defibrillator deployment. ${ }^{6}$

There were some important limitations to this study. Firstly, this was a retrospective singlecenter study and may not adequately reflect the disease burden in other areas of the world. Secondly, as this study focused exclusively on clinical disease, the presence of subclinical cardiac dysfunction was not assessed, although the significance of subclinical cardiac disease is highly debated. ${ }^{2,7}$ Lastly, while our cohort fortunately did not demonstrate acute coronary abnormalities, this study does not seek to determine the long-term risk of coronary artery involvement in children with mild COVID-19 infections. While the evidence regarding coronary artery involvement has mostly focused on children with either severe COVID-19 infection or MIS-C, additional study should be performed in the pediatric patients with less severe infection to determine if they need ongoing cardiac surveillance. $^{8}$

In closing, school-age children who were not hospitalized for COVID-19 have a very low risk of having clinically significant cardiac damage and are more likely to discover incidental findings. We hope our experience provides context with regard to the need for cardiac testing in the nonhospitalized, asymptomatic school-aged children.

\section{REFERENCES}

1. American Academy of Pediatrics. COVID-19 interim guidance: return to sports and physical activity. Updated March1,2021. Accessed May 13,2021. Availablein:https: / / services.aap.org/en / pages / 2019-novel-coronaviruscovid-19-infections / clinical-guidance / covid-19-interimguidance-return-to-sports /

2. Moulson N, Petek BJ, Drezner JA, Harmon KG, et al. SARSCoV-2CardiacInvolvementin Young Competitive Athletes. Circulation. 2021;144(4):256-66.

3. Dyal J, Schwartz AD, Oster M, Mahle W. Cardiac screening after COVID-19 infection in children: is electrocardiogram warranted? J Am Coll Cardiol. 2021 May 11;77(18):3184.

4. Zimmermann $\mathrm{P}$, Curtis N. Coronavirus Infections in Children Including COVID-19: An Overview of the Epidemiology, Clinical Features, Diagnosis, Treatment and Prevention Options in Children. Pediatr Infect Dis J. 2020;39(5):355-68.

5. Gartenberg AJ, White TJ, Dang K, Shah M, et al. Assessing the Utility of Screening Electrocardiograms in Pediatric Patients Following COVID-19. Cardiol Young. 2021:1-7.

6. DreznerJA, ToresdahlBG, Rao AL, HusztiE, etal. Outcomes from sudden cardiac arrest in US high schools: a 2-year prospective study from the National Registry for AED Use in Sports. Br J Sports Med. 2013;47(18):1179-83.

7. Brito D, Meester S, Yanamala N, Patel H, et al. High Prevalence of Pericardial Involvement in College Student Athletes Recovering From COVID-19. JACC Cardiovasc Imaging. 2021;14(13):541-55.

8. Feldstein LR, Tenforde MW, Friedman KG, Newhams M, et al. Overcoming COVID-19 Investigators. Characteristics and Outcomes of US Children and Adolescents With Multisystem Inflammatory Syndrome in Children (MIS-C) Compared With Severe Acute COVID-19. JAMA. 2021;325(11):1074-87. 